\title{
Theory of Laser Energy Harvesting at Femtosecond Scale
}

\author{
Vladimir L. Kalashnikov \\ Additional information is available at the end of the chapter \\ http://dx.doi.org/10.5772/intechopen.75039
}

\begin{abstract}
Energy scaling of femtosecond laser pulses has a lot of applications in nanoscale micromachining, precision time-resolution spectroscopy, high-harmonic generation, surgery, etc. Besides applied sciences and technology, there are fundamental applications of energy harvesting at femtosecond scale. In particular, it is possible to study and control intra-atom and molecular dynamics at attosecond level as well as to map the quantum processes directly with unprecedented spatial and temporal resolution. This "mesoscopic" union of classical and quantum phenomena provides with new insights into fundamental issues of quantum mechanics of open systems including possible application in the field of quantum computing. In this work, we consider a theory of femtosecond pulse energy harvesting using the dissipative soliton generation in both solid-state and fiber mode-locked lasers and the femtosecond pulse enhancement in an external resonator. The femtosecond pulse energy, width, and spectrum scaling laws are presented in the explicit and physically meaningful form.
\end{abstract}

Keywords: mode-locked laser, dissipative soliton, external enhancement resonator, femtosecond pulse energy scaling, spectral extra-broadening

\section{Introduction}

In the last decades, the breakthrough in the energy scalability of femtosecond laser pulses has been achieved that bring high-energy physics on tabletops of a mid-level university lab [1, 2]. As a result, the intensities of $\sim 10^{15} \mathrm{~W} / \mathrm{cm}^{2}$ become available directly from a mode-locked thin-disk laser oscillator operating at an over-MHz repetition rate [3-8]. Such systems are 
considerably more simple, functional and economical than classical chirped-pulse amplifiers (of course, at the $\sim \mathrm{GW}$-pulse power level) $[9,10]$. Moreover, a high repetition rate provides the signal/noise ratio improvement of $10^{3}-10^{4}$ in comparison with an ordinary $\mathrm{kHz}$ chirped pulse amplifier. In practice, such oscillators are of interest for direct gas ionization and highharmonic generation [11, 12], pump-probe diffraction experiments with electrons [13] and fabrication of fine structures in transparent and semi-transparent materials [14], characterization and control of the electronic dynamics, metrology and ultra-sensitive spectroscopy, biophotonics and biomedicine, etc. $[2,15,16]$.

The phenomenon of ultrashort pulse energy harvesting exceeds the limits of immediate laserbased applications and is involved in a much broader context of formation and control of macroscopic coherent structures [17]. The high-energy ultrafast lasers become an excellent tool for testing the fundamental problems of self-organization and nonlinear dynamics far from thermodynamic equilibrium which cover the area ranging from hydrodynamics to condensed matter physics and even biology and sociology [18-20]. Such an approach based on the transfer of issue of complicate dynamics to another simpler material context can be named "metaphoric" or "analog" modeling [21, 22] and successes due to high controllability, relative simplicity, and unique potential of statistic gathering inherent in lasers systems [1].

The idea of energy $E$ harvesting is based on an elementar relation: $E=P_{a v} T_{r e s}$, where $P_{a v}$ is an average power in a resonator with an effective period $T_{\text {res }}$. Scaling of $P_{a v}$ and/or $T_{\text {res }}$ would provide the scaling of ultrashort pulse energy on condition that a stable ultrashort pulse emerges spontaneously (so-called, mode-locking self-start condition) in a laser system. As will be shown, these conditions are highly non-trivial for energy-scalable lasers and can limit substantially the pulse energy and its width. Two main approaches to the energy harvesting at femtosecond scale will be considered in this work.

The first one is based on the unique capacity of laser dissipative solitons (DS) [17, 23] to accumulate an energy without loss of stability [24, 25]. Some basic approaches to study of the energy-scaling laws for such systems will be presented, and the limits of energy and pulse width scalability will be outlined.

The second approach is based on the energy storing in an external high-Q resonator (so-called enhancement resonator, ER) coupled synchronously with a femtosecond pulse oscillator [26-28]. This simple idea faces difficulties when it is realized on a femtosecond scale because nonlinear effects and group-delay dispersion (GDD) tend to destroy a synchronization between a laser and ER. These issues will be outlined, and some modifications of ER technique will be proposed.

\section{DS energy scaling}

The DS energy and width scaling are connected closely with a duality between amplification of the maximum number of laser modes and simultaneous spectral condensation, i.e., the concentration 
of energy within the strongly confined spectral region. It is important that all excited and amplified modes must be phase-synchronized, i.e., coherent.

In a trivial model of laser, there exists a set of $N$-longitudinal resonator modes which are separated by the frequency interval of $\delta \omega=2 \pi / T_{\text {res }}$ and excited by a gain medium with the gain-bandwidth $\Omega: N \sim \Omega / \delta \omega$ [29]. These modes are mutually phase-independent and incoherent, and a result of their interference $A=\sum_{n=-N / 2}^{N / 2} a_{i} \cos \left[\left(\omega_{0}+n \delta \omega\right) t+n \delta \phi\right]$ is the irregular field beatings with the width of separated spikes $\sim 1 / N \delta \omega=1 / \Omega$ (Figure 1(a); the intermode phase difference $\delta \phi$ is random). However, a fixed inter-mode phase difference results in regular spikes of the $\sim 1 / \Omega$ width with the peak power $\sim N^{2}$ and the repetition-period $=T_{\text {res }}$ (Figure 1(b)) [30]. The last phenomenon is called mode-locking (ML) and underlies a coherent energy condensation within short-time intervals. Respectively, the spectral width of each spike tends to $\Delta \Omega$.

However, this simple scheme faces many complications. Well, to be precise, a gain-band is not uniform (bell-shape like) and a mode, which is closest to a frequency $\omega_{0}$ at gain maximum, has maximum amplification. Since laser gain is energy-saturable, this mode concentrates all energy and suppresses the competitive modes. This is a mode selection process. Therefore, a multimode generation leading to ultrashort pulse formation is not a genuine but emergent phenomenon which requires a multimode instability.

There are several possible mechanisms for such instability [31] which are closely connected with the issue of the ML self-start. Existing theories of the ML self-start predict a lot of effects involved in a laser pulse formation including mode-beatings [32, 33] and hole burning, induced refractive grating in an active medium [34], dynamic gain saturation [33, 35], parasitic reflections and absorption in a resonator [36], continuous-wave instability [37, 38] and Risken-Nummedal-Graham-Haken effect [39]. The thermodynamic theory of ML self-start has been developed, and it has been shown that the pulse appearance is a first-order phase transition, which is affected strongly by the laser noises distributed over a whole resonator period [40-42]. In any case, a stable ML requires whether a nonlinear resonant excitation by
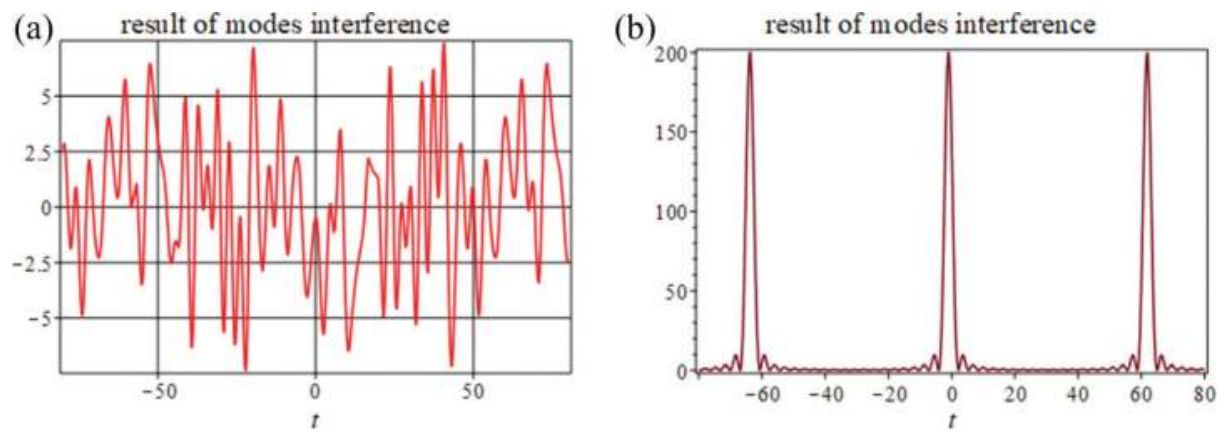

Figure 1. Interference of phase uncoupled (a) and locked (b) modes $(N=20)$ [30]. 
external periodic "force" (active mode-locking) ${ }^{1}$ or a mutual mode coupling through an optical nonlinearity (passive mode-locking). The excitation of harmonics at $\pm n \delta \omega$ couples and synchronizes the adjacent modes and provides their phase-locking.

The mechanisms of ML are beyond the scopes of this work, and we will focus on the principles of the sustained ML energy-scalable regimes at femtosecond scale. The basic principle under consideration is to exploit DS [23] which is extremely stable in nonequilibrium dissipative environment [24, 25]. Since DS behaves like a soliton of integrable systems [17, 44], its dynamics can be described by some distributed nonlinear model. The most famous and studied one is based on the complex nonlinear Ginzburg-Landau equation which can be treated as a dissipative extension of the nonlinear Schrödinger equation [45]. ${ }^{2}$

A very productive approach to the study of this class of equations is based on the so-called variational approximation (VA) [49-51]. The non-dissipative effects can be described by the Lagrangian density:

$$
L=\frac{i}{2}\left(a^{*}(z, t) \mathrm{\partial}_{z} a(z, t)-a(z, t) \mathrm{\partial}_{z} a^{*}(z, t)\right)-\frac{1}{2} \gamma|a(z, t)|^{4}+\frac{\beta_{2}}{2} \partial_{t} a(z, t) \mathrm{\partial}_{t} a^{*}(z, t),
$$

where $a(z, t)$ is a complex slowly varying field amplitude, $t$ and $z$ are local time and propagation distance, respectively, $\gamma$ is a self-phase modulation (SPM) coefficient and $\beta_{2}$ is a groupdelay dispersion (GDD) coefficient. The Euler-Lagrange equation corresponding to Eq. (1) is the nonlinear Schrödinger equation:

$$
i \partial_{z} a(z, t)=\frac{\beta_{2}}{2} \partial_{t, t} a(z, t)+\gamma|a(z, t)|^{2} a(z, t) .
$$

Further, two different types of DS will be considered: (i) chirped-free

$$
a(z, t)=\alpha(z) \operatorname{sech}(t / T(z)) \exp (i \phi(z))
$$

and (ii) chirped pulses

$$
a(z, t)=\alpha(z) \operatorname{sech}(t / T(z))^{1+i \psi(z)} \exp (i \phi(z))
$$

where $\alpha, T, \psi$ and $\phi$ are DS amplitude, width, chirp, and phase-delay, respectively.

\subsection{Chirped-free DS}

VA in action looks like following. Substitution of the trial solution (3) into (1) with the subsequent integration over $t$ results in the reduced Euler-Lagrange equations [52]:

\footnotetext{
${ }^{1}$ This phenomenon is closely related to the concept of stochastic resonance which describes processes of resonant coherence enhancement in a noisy periodically driven system [43].

'Different versions of this equation describe an extremely broad area of phenomena ranging from laser dynamics [17, 46, 47], oscillatory chemical reactions [22] to Bose-Einstein condensations and biological systems [48].
} 


$$
\begin{gathered}
4 \gamma \alpha^{2}+6 \partial_{z} \phi=\beta_{2} / T^{2}, \\
\beta_{2} / T^{2}+2\left(\gamma \alpha^{2}+3 \partial_{z} \phi\right)=0, \\
2 \alpha^{2} \partial_{z} T+4 T \alpha \partial_{z} \alpha=0 .
\end{gathered}
$$

Eq. (7) is the energy conservation law, and Eqs. (5) and (6) give the parameters of Schrödinger soliton if $\partial_{z} T=0, \partial_{z} \alpha=0$ :

$$
\alpha T=\sqrt{\beta_{2} / \gamma}, q \equiv-\partial_{z} \phi=\frac{\beta_{2}}{2 T^{2}}
$$

where the first expression is the soliton area theorem, and the last one defines the soliton wavenumber $q$.

\subsubsection{Perfectly saturable absorber}

The effect of dissipative factors can be taken into account using the Ritz-Kantorovich method [51-54] when the reduced Euler-Lagrange equations are driven by a dissipative "source" [52]:

$$
\begin{aligned}
& \frac{d}{d z} \frac{\partial \int L d t}{\partial\left(b_{i}\right)_{z}}-\frac{\partial \int L d t}{\partial b_{i}}=2 \operatorname{Re} \int Q \frac{\partial a^{*}}{\partial b_{i}} d t \\
& Q=-i \Gamma a+\frac{i \rho\left(a+\tau \partial_{t, t} a\right)}{1+\sigma \int|a|^{2} d t}+\frac{i \mu \zeta|a|^{2} a}{1+\zeta|a|^{2}} .
\end{aligned}
$$

Here the reduced Lagrangian $\int L d t$ is calculated using a trial function (i.e., Eq. (3)) with the parameters $b_{i}=\{\alpha, T, \phi\}$. The dissipative factors are defined by a net-loss with the coefficient $\Gamma$, a small-signal gain $\rho$ with the inverse saturation energy $\sigma$ and a squared inverse gain bandwidth $\tau$. The self-amplitude modulation (SAM) providing ML is described by an effective "perfectly saturable absorber" [24] with the modulation depth $\mu$, and the inverse saturation power $\zeta$.

A solution obtained from Eqs. (3) and (9) gives the expressions for area theorem and phase delay corresponding to the Schrödinger soliton. But the DS amplitude is defined by dissipative factors [52]:

$$
\frac{\mu \log \left(\frac{1+\alpha^{2}-\alpha \sqrt{1+\alpha^{2}}}{1+\alpha^{2}+\alpha \sqrt{1+\alpha^{2}}}\right)}{\alpha \sqrt{1+\alpha^{2}}}+2(\Sigma+\mu)-\frac{2}{3} C \alpha^{2}=0,
$$

where $\Sigma=\frac{\rho}{1+2 \alpha^{2} T \sigma}-\Gamma, C=\frac{\rho \tau \gamma}{\beta_{2} \zeta}\left(1+2 \alpha^{2} T \sigma\right)^{-1}$. The peak power $\alpha^{2}$ in Eq. (10) is normalized to $\zeta$. It is convenient to assume that a laser operates in the vicinity of a threshold in steady-state regime: $\frac{\rho}{1+2 \alpha^{2} T \sigma} \approx \Gamma$ and $\Sigma \approx 0, C \approx \frac{\Gamma \tau \gamma}{\beta_{2} \zeta}$. 
The marginal stability condition $\Sigma=0$ defines a stability of DS against continuous-wave or multiple pulse generation [30,55], and the DS approaching this stability threshold has a minimum width and a best asymptotical energy scalability $\left(E=2 \alpha^{2} T \zeta / \sqrt{\tau \Gamma} \gg 1\right)$ [52]:

$$
\begin{aligned}
& C \rightarrow \text { const } \times \sqrt{\mu \tau \Gamma} / E \zeta, E \rightarrow \text { const } \times \frac{\beta_{2}}{\gamma} \sqrt{\frac{\mu}{\tau \Gamma^{\prime}}} \\
& \alpha^{2} \rightarrow\left(\frac{\text { const }}{2}\right)^{2} \frac{\mu \beta_{2}}{\tau \Gamma \gamma}, T \rightarrow \frac{2}{\text { const }} \sqrt{\frac{\tau \Gamma}{\mu}}, \text { const } \approx 3.535 .
\end{aligned}
$$

These scaling laws demonstrate main principles of chirped-free pulse energy harvesting. Of course, these dependencies can be considered as only qualitative ones. Nevertheless, they demonstrate that the asymptotic DS energy scales $\alpha \beta_{2}$, and the minimum pulse width is defined by not only the medium gain bandwidth $\propto 1 / \sqrt{\tau}$ but the net-loss $\Gamma$ and the modulation depth $\mu$, as well (Figure 2).

Thus, the pulse can be squeezed by scaling of modulation depth with a parallel decrease of the stabilizing dispersion. Additional pulse shortening can be provided by net-loss lowering (see Figure 2). These tendencies are quite reasonable because the selective spectral properties of an active medium are defined by not the gain for a small signal but by the saturated gain $\approx \Gamma$ near the pulse stability threshold (i.e., since no gain, no gain induced spectral selection). Simultaneously, the modulation depth defines an inter-mode coupling strength that favors ML and, thereby, pulse spectrum broadening with $\mu$-growth.

Eqs. (8) and (10) demonstrate that an approach to the threshold $C(\Sigma \rightarrow 0)$ as well as a higher $E \propto \zeta / \sqrt{\tau}$ minimize the pulse width $T$ (Figure 3).

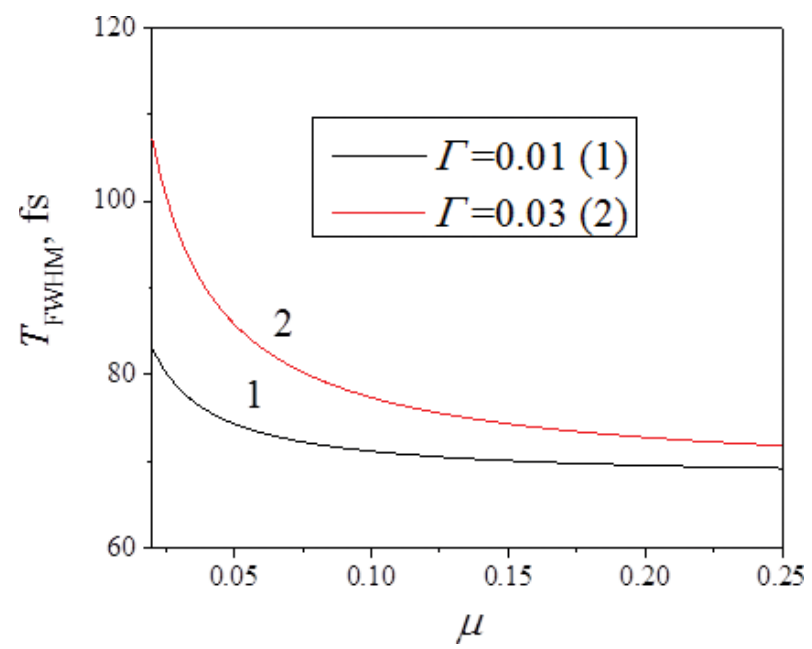

Figure 2. Dependence of the asymptotic full width at half maximum (FWHM) $T_{\text {FWHM }}$ on the modulation depth $\mu$ for different net-loss coefficients $\Gamma$. The gain bandwidth of $5.3 \mathrm{THz}$ corresponds to a Yb: YAG thin-disk active medium. 

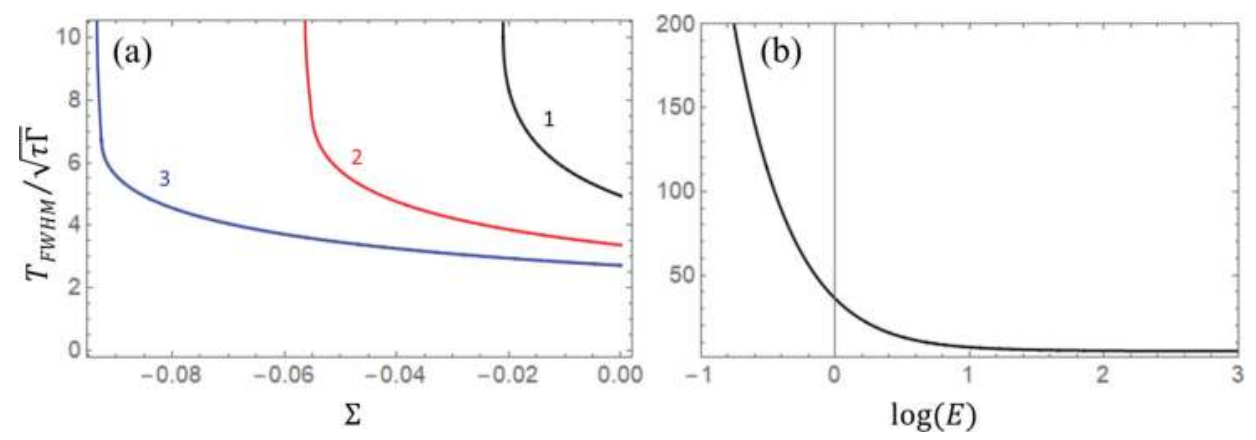

Figure 3. Dependence of the normalized $T_{\mathrm{FWHM}}$ on $\Sigma$ (a) for $\mu=0.05$ (1), 0.1 (2), 0.15 (3), $C=0.01$; and on the normalized energy $E(b)$ for $\mu=0.05, \Sigma=0$.

Properties of DS are described by the so-called master diagram [24] which represents the DS parametric space and is shown in Figure 4(a). There are two DS-solutions of Eqs. (1), (3), (9) and (10): (i) upper branch (i.e., the branch with the larger $C$ for fixed $E$ and $\Sigma$, see Figure 4(a)) corresponds to the above considered energy-scalable DS. The energy scalability for this type of soliton is accompanied by minimization of its width (Figures $\mathbf{3}$ and $\mathbf{4}(\mathbf{b})$; i.e., $\lim _{E \rightarrow \infty, \Sigma \rightarrow 0} T=$ $\frac{2}{\text { const }} \sqrt{\frac{\tau \Gamma}{\mu}}$, see Eq. (11)) and, respectively, by the growth of peak power. Namely, this branch has a threshold of marginal stability $\Sigma=0$ (Figure 4(a); curve 1). (ii) lower branch (Figure 4 (a)) corresponds to a DS energy scalability provided by its width growth (Figure 4(b); $\left.\lim _{E \rightarrow \infty, \Sigma<0} T=\infty\right)$. Thus, this DS branch is unpractical for energy scaling because the broad chirp-free pulse would require an additional nonlinear mechanism for external compression. One can name this branch as energy-unscalable.

A fundamental property of the DS solutions presented is their stability. The VakhitovKolokolov stability criterion $d E / d q>0[56,57]$ demonstrates the stability of both branches of DS (see Eq. (8)):
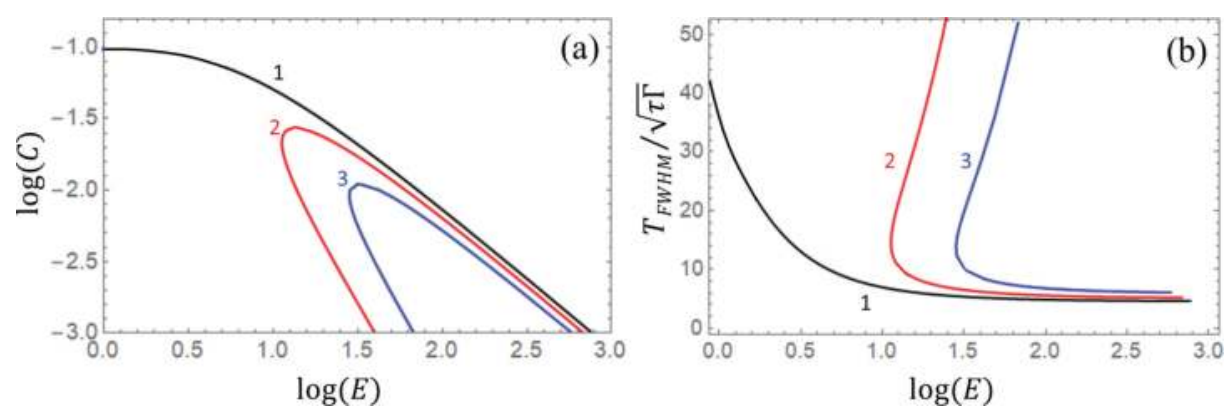

Figure 4. Master diagram (a) and the corresponding DS widths (b). $\Sigma=0$ (1), -0.01 (2), and -0.02 (3), $\mu=0.05$. 

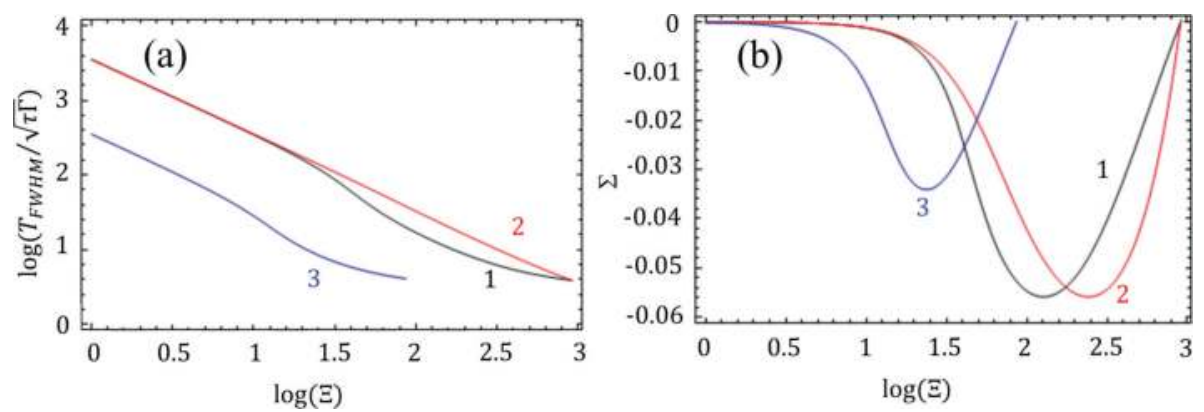

Figure 5. The dimensionless DS widths (a) and the corresponding net-gain (b) in dependence on the threshold energy $\Xi$ for $\delta=-0.05, C=10^{-3}(1) ; \delta=-0.5, C=10^{-3}(2)$; and $\delta=-0.05, C=10^{-2}(3) . \mu=0.07$.

$$
\frac{d E}{d q}=\frac{d}{d q}\left(2 \alpha^{2} T\right)=\frac{d}{d q}\left(2 \sqrt{2 \beta_{2} q} / \gamma\right)=\frac{\sqrt{2 \beta_{2} / q}}{\gamma}>0
$$

It means physically that an energy scalability of DS does not suffer from soliton collapse and both DS branches are feasible.

As was mentioned above, the net-gain $\Sigma$ is energy-dependent, and such a dependence has to be taken into account. In the neighborhood of the laser threshold where $\Sigma(E) \approx 0$, one may expand the net-gain coefficient near a threshold energy $\Xi=\sigma^{-1}(\rho / \Gamma-1)$ :

$$
\Sigma=\left.\frac{d}{d E}\left(\frac{\rho}{1+\sigma E}-\Gamma\right)\right|_{E=\Xi}+O\left(\frac{d^{2}}{d E^{2}}\right) \approx \delta\left(\frac{E}{\Xi}-1\right)
$$

where $\delta=-\Gamma^{2} \Xi \sigma / \rho$

Figure 5(a) shows the DS dependence on the threshold energy for a fixed control parameter $C$ in the presence of gain saturation. DS squeezes with energy, and such a squeezing is confined by the stability criterion $\Sigma<0$. Simultaneously, $\Sigma$ decreases from 0 with energy (Figure 5(b)), that corresponds to the "energy unscalable" DS, with the subsequent growth up to 0 , that corresponds to the "energy scalable" DS. Thus, there is not a "switch" between two different types of DS in a real-world laser system which behaves quite smoothly with energy.

\subsubsection{Cubic-quintic SAM}

Physically, this type of SAM describes approximately an action of nonlinear polarization rotation, which is a typical ML mechanism for fiber lasers, or a so-called "soft aperture" Kerrlens ML typical for solid-state lasers [24, 25]. In this case, loss saturation switches to the loss growth at $\alpha^{2}=1 / 2 \zeta$ and

$$
Q=-i \Gamma a+\frac{i \rho\left(a+\tau \partial_{t, t} a\right)}{1+\sigma \int|a|^{2} d t}+i \kappa\left(1-\zeta|a|^{2}\right)|a|^{2} a .
$$

The $\kappa$-parameter plays a role of the inverse loss saturation power, and the modulation depth is $\mu=\kappa / 4 \zeta$. 
The solutions for T and $q$ correspond to Eq. (8), but the two-branch solution for DS peak power can be expressed in an explicit form:

$$
\alpha^{2}=\frac{5}{16}\left(2-C \mp \sqrt{(2-C)^{2}+96 \zeta \Sigma / 5 \kappa}\right)
$$

(power, time and energy are normalized to $\zeta \sqrt{\kappa / \zeta \tau \Gamma}$ and $\sqrt{\kappa \zeta / \tau \Gamma}$, respectively, and $\left.C=\tau \Gamma \gamma / \beta_{2} \kappa\right)$. These branches are separated by the energy curve $E=\sqrt{5(2-C) / C} / 2$ (curve 1 in Figure 6), and are shown in Figure 6.

The asymptotic scaling laws for this type of SAM are:

$$
\begin{aligned}
& C \rightarrow \frac{5 \tau \Gamma}{4 \mu \zeta^{2} E^{2}}, E \rightarrow \sqrt{\frac{20 \mu \beta_{2}}{\kappa \gamma}}=\sqrt{\frac{5 \beta_{2}}{\zeta \gamma^{\prime}}} \\
& \alpha^{2} \rightarrow \frac{5 \mu}{\kappa}, T \rightarrow \sqrt{\frac{\kappa \beta_{2}}{5 \gamma \mu} .}
\end{aligned}
$$

One can see that the energy scaling is provided by the DS width (not power) scaling that is a natural consequence of the peak power confinement $\alpha^{2}=1 / 2 \zeta$ imposed by a SAM saturation ${ }^{3}$. Both branches behave quite congruently in this case (Figure 6).

\subsubsection{Energy harvesting of the chirp-free DS at femtosecond scale}

Most promising devices realizing the femtosecond-pulse energy scalability are thin-disk solidstate lasers $[5,6,24,58]$ which provide an excellent average power scaling and controllable nonlinear effects limiting the energy scalability in fiber oscillators [25]. Nevertheless, there are some main obstacles for further energy harvesting at femtosecond scale for such a type of

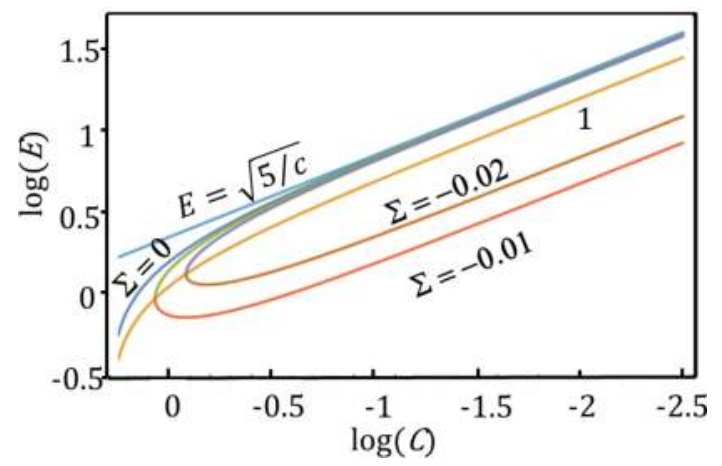

Figure 6. Master diagram for the chirp-free DS and the cubic-quintic SAM. Curve 1 divides two different branches of DS. The upper line $E=\sqrt{5 / C}$ corresponds to asymptotical energy scaling law. ${ }^{3}$ In the case of perfectly saturable SAM, the confinement is imposed by spectral dissipation (i.e., the DS width but not
power is confined; see Eq. (11)). 
devices. (i) a traditional ML mechanism uses the structured semiconductor devices (so-called semiconductor saturable absorber mirrors, SESAM) which $(\alpha)$ have a slow ( 100 fs) response time; $(\beta)$ a complicated and hardly-controllable kinematics including higher-order nonlinear effects, non-saturable losses, temperature and radiational damage, etc.; $(\gamma)$ SAM and SPM effects are decoupled for this type of ML that requires growth of GDD for DS stabilization in accordance with the area theorem (Eq. (8)). (ii) As was shown (Eq. (11)), the minimum pulse width is $\propto \sqrt{\tau \Gamma / \mu}$ so that using the media with the broader gain band would provide a pulse shortening down to sub-100 fs [59]. However, such media with good optical quality are not widely available and technologically advanced.

Nevertheless, an alternative approach to energy scalability of femtosecond pulses has been demonstrated in [7]. (i) ML mechanism can be provided by an instantaneous self-focusing (Kerr-lensing) induced by a set of nonlinear crystals inside a laser resonator. (ii) Such a mechanism combines both SAM and SPM that enhances the SAM parameters ( $\mu$ and $\zeta$ ) in parallel with the SPM one ( $\gamma$, see Eq. (11)). As a result, the GDD value can be reduced in parallel with the DS shortening in agreement with the area theorem. (iii) A real-world gain band profile is Lorentzian, not Gaussian as in Eqs. (9) and (14).

The Lorentzian gain profile can be taken into account by using the numerical simulations of the generalized complex nonlinear Ginzburg-Landau equation [60, 61]:

$$
\mathrm{\partial}_{z} a=-\Gamma a+\frac{\mu \zeta|a|^{2}}{1+\zeta|a|^{2}} a-i\left(\frac{\beta_{2}}{2} \partial_{t, t} a+\gamma|a|^{2} a\right)+\frac{\rho \Omega}{2\left(1+\sigma \int|a|^{2} d t\right)} \int_{-\infty}^{t} a\left(z, t^{\prime}\right) \exp \left[-\Omega\left(t-t^{\prime}\right)\right] d t^{\prime},
$$

where a characteristic gain bandwidth is $\Omega \propto 1 / \sqrt{\tau}$.

As was shown in [60], the Lorentzian gain profile gives more efficient amplification and broader spectrum than the Gaussian one. Additionally, an inherent gain dispersion shifts the DS spectrum and affects its shape [62]. The numerically obtained pulse spectra for different modulation depths are shown in Figure 7. One can see a pronounced spectrum broadening and, correspondingly, pulse shortening with the modulation depth growth.

The dependences of minimum DS width and corresponding stabilizing GDD on the modulation depth for different values of the SAM saturation power $\zeta$ are shown in Figure 8. One can see that the DS shortens with $\mu$ in qualitative agreement with the analytical results presented above. Simultaneously, the DS spectrum is noticeably broader than the gain band that provides a generation of sub-50 fs pulses at the MW peak power level directly from an oscillator. Since a pulse is chirp-free, the threshold stabilizing anomalous GDD decreases, as well, in agreement with the soliton area theorem. Simultaneously, there is the nonmonotonic dependence of DS width on the SAM parameter $\zeta$ so that $T$ decreases initially and then increases with the $\zeta$-decrease, i.e., with the saturation power growth. The growth of saturation power ( $\zeta$-decrease) causes a threshold-like increase of pulse width and stabilizing GDD for small modulation depths $\mu$. 


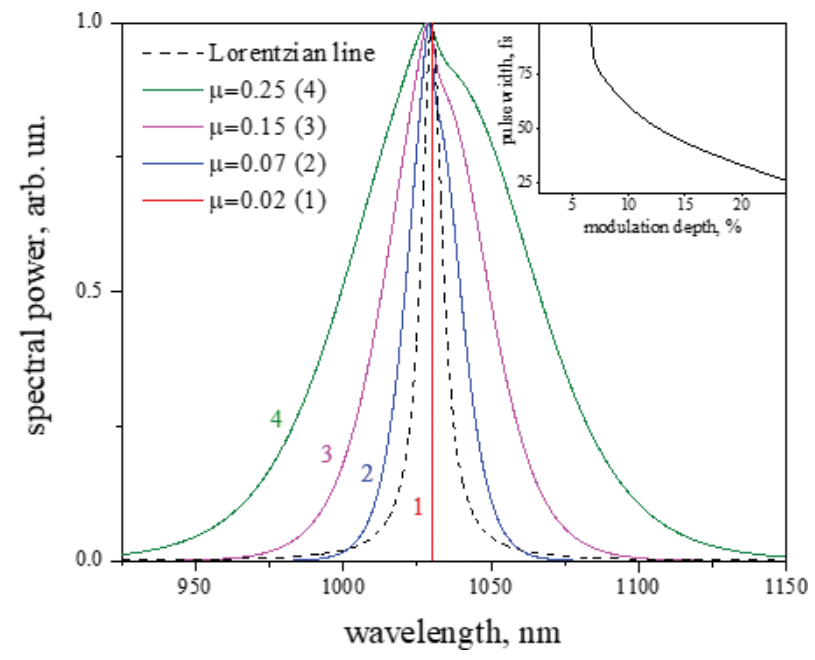

Figure 7. Numerical spectral profiles for different modulation depths $\mu$ (inset shows the dependence of $T_{\text {FWHM }}$ ). GDD corresponds to the stability threshold, $\zeta=\gamma=1.35 \mathrm{MW}^{-1}, \delta=-0.05$. The Lorentzian gain band of $5.3 \mathrm{THz}$ (dashed line) corresponds to a $\mathrm{Yb}$ : YAG, the output energy $E_{\text {out }}$ is of $\approx 0.011 \mu \mathrm{J}$ for $3 \%$ output coupler.
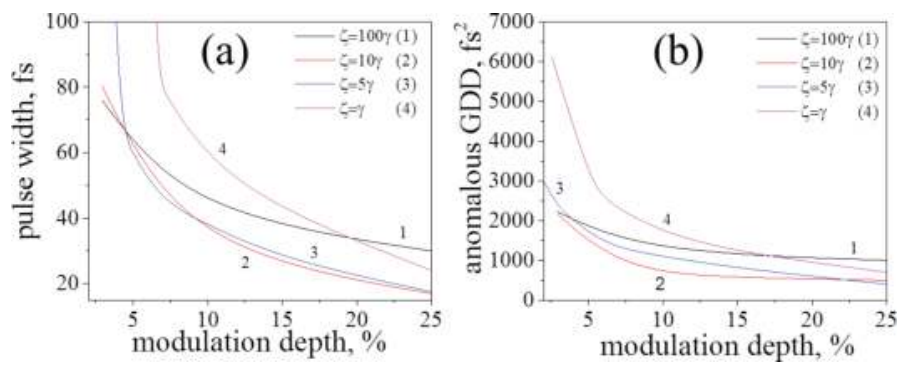

Figure 8. Pulse width $T_{F W H M}$ vs. modulation depth (a) along the boundary GDD (b) in dependence on the saturation parameter $\zeta$. $E_{\text {out }} \approx 0.011-0.014 \mu \mathrm{J}$ and other parameters correspond to Figure 7.

Figure 9 illustrates the energy-dependence of the DS width and the stabilizing GDD for a low net-loss. The short pulses are possible in this case, as well, but a small modulation depth does not allow a substantial DS shortening.

\subsection{Chirped DS}

Chirped DS demonstrates a high potential for energy harvesting in both solid-state and fiber lasers [24, 25] due to enhanced stability provided by well-structured energy redistribution inside a pulse. An energy scalability results from the DS stretching that limits its peak power 
and, thereby, suppresses an instability caused by nonlinearity. This factor is especially important for all-fiber lasers, where the strong contribution of nonlinear effects is inevitable with $T_{\text {res }}$-growth. VA predicts the following energy-scaling laws ${ }^{4}$ :

$$
E \propto\left|\beta_{2}\right| / C \sqrt{\tau \Gamma}, E \propto\left|\beta_{2}\right| / \sqrt{\tau \Gamma}
$$

for the SAM described by Eqs. (9) and (14), respectively [24, 25].

The chirped DS accumulates energy $\propto \psi$ that allows using a so-called adiabatic theory for $\psi \gg 1$ $[24,63,64]$ which predicts a perfect energy scalability or a DS resonance [65] for the cubicquintic SAM (Eq. (14)). That means that energy can be scaled infinitely for $C=1 / 3$ due to pulse stretching and simultaneous spectral condensation:

$$
\lim _{C \rightarrow 1 / 3}\left\{\begin{array}{l}
E \rightarrow \infty \\
\alpha^{2} \rightarrow 1 / \zeta \\
\Delta \rightarrow \sqrt{2 \gamma / \beta \zeta} \\
\Theta \rightarrow 0 \text { ("spectral condensation") }
\end{array},\right.
$$

where the DS spectral profile is described by a truncated Lorentzian function:

$$
p(\omega)=\frac{6 \pi \gamma}{\kappa \zeta} \frac{H\left(\Delta^{2}-\omega^{2}\right)}{\omega^{2}+\Theta^{2}}
$$

(here $H$ is the Heaviside's function).

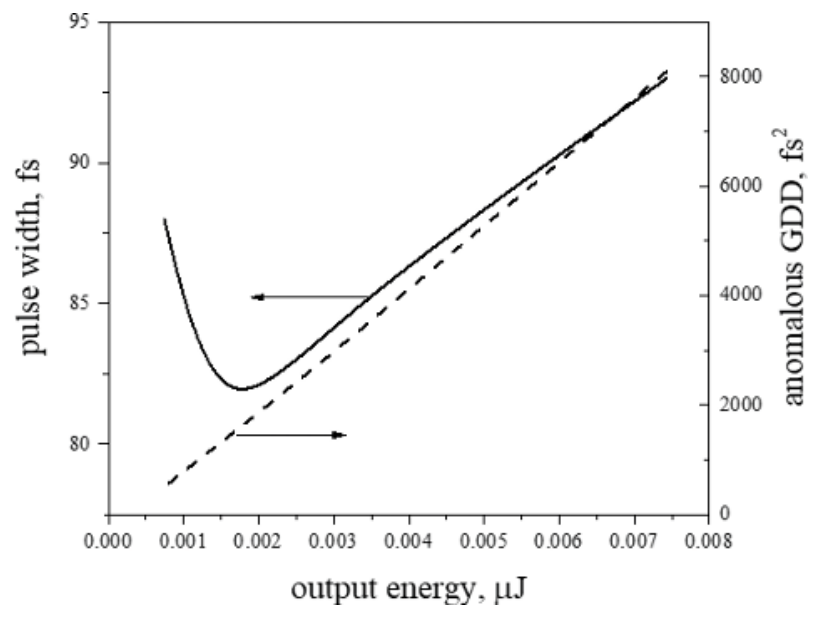

Figure 9. A low-loss regime with $\delta=-0.05 / 3, \zeta=100 \gamma, 1 \%$ output coupler, $\mu=2 \%$.

\footnotetext{
${ }^{4}$ The negative sign of $\beta_{2}$ corresponds to a normal GDD in these notations.
} 
For the SAM presented by Eq. (9), the adiabatic theory predicts the energy-scaling law in the form of $[66]^{5}$ :

$$
E \propto \frac{\left|\beta_{2}\right|}{\zeta \sqrt{\tau}}\left[\frac{3}{C} \sqrt{1-C}+\operatorname{arctanh}(\sqrt{1-C})\right]
$$

that gives the first expression in Eq. (18) in the $C \rightarrow 0$ (i.e., $E \rightarrow \infty$ ) limit.

Since the chirped DS energy scaling is provided by its stretching $\propto \psi$, this process is reversible so that an output DS can be compressed by a factor $\approx 1 / \psi$. Nevertheless, some energy loss occurs with such compression due to nonuniformity of DS chirp [68] $]^{6}$ that requires optimizing the DS and pulse compressor parameters.

\subsection{Main obstacles to the DS energy harvesting}

The DS energy harvesting in both chirped and chirp-free regimes has a common problem of $M L$ self-starting. The DS stability is a necessary but not sufficient condition of its existence because it must develop from some stochastic process in a laser (eventually, from a quantum noise). Existing theories of the ML self-start [32-42] predict that a lot of effects are involved in a pulse formation. However, a spontaneous formation of the DS from noise (the DS self-start) as a general problem has not been studied in depth. In optics, this is often considered as a technical issue, because here one can use one of the proven ML techniques to guarantee self-starting. After the initial kick, however, the DS evolves by itself, and recent experiments have shown controversial results: in high-power solid-state lasers, the strong oscillations ( $Q$-switching) during the DS buildup dynamics hinders the DS self-start [69], while in a fiber laser, such oscillations can accelerate the self-start [70]. That is obviously connected to co-existence of nonlinearities with different time scales: instantaneous non-dissipative SPM, and non-instantaneous dissipative nonlinearities like stimulated Raman scattering (SRS), saturable absorber losses, and gain saturation. This issue is especially intriguing, as the dynamic gain saturation can provide a supplementary mechanism of DS formation [71].

The growing nonlinearity results in quite nontrivial modification of dynamics [25] and causes whether DS stabilization or its chaotization [72-74]. For example, the practically relevant Yb-based thin-disk lasers possess reduced instantaneous nonlinearity and longer gain relaxation times as compared to a bulk Ti: sapphire laser. In the latter case, the enhanced dynamic gain saturation can destabilize a much-desired high-energy DS [75-77]. As another example, the experiments demonstrated, that DS energy scaling in all-fiber fiber lasers is limited by energy loss due to SRS [78]. Nevertheless, SRS could play a positive role providing the generation of dissipative Raman soliton and suppressing the optical turbulence [79-81]. The connection of this phenomenon to the general issues of the turbulence theory waits for its exploration [82].

\footnotetext{
${ }^{5}$ The adiabatic theory does not predict a spectral condensation near the carrier frequency $\omega \approx 0$ for this SAM law, but such a concentration is possible at spectrum edges. This phenomenon is clearly visible in the numerous experiments and can be explained by the DS perturbation theory [67].

${ }^{6}$ That is a measure of the DS fidelity.
} 


\section{Femtosecond pulse enhancement in an external resonator}

Using a high-finesse ER coupled with a mode-locked femtosecond laser is the method of energy storing allowing broadband absorption spectroscopy [83], high-harmonic generation and frequency comb generation up to the extreme ultraviolet frequency [84, 85].

Equations describing a coupling with ER are [86, 87]:

$$
b_{r}=r b_{i n}+\theta a_{i n}, \quad a_{r}=\theta b_{i n}-r a_{i n}
$$

where $b_{r}$ and $b_{i n}$ are the reflected and incident fields on the coupler from a side of femtosecond oscillator; $a_{r}$ and $a_{i n}$ are the corresponding fields from a side of ER; and $\theta=\sqrt{1-r^{2}}, r$ are the transmittance and reflection coefficients of a coupler, respectively. The field inside ER evolves as:

$$
a_{i n}(t)=\exp \left[-\Gamma+i \phi-\beta_{1} \frac{\partial}{\partial t}+i \sum_{m=2}^{N} i^{m} \beta_{m} \frac{\partial^{m}}{\partial t^{m}}+i \gamma\left|a_{r}(t)\right|^{2}\right] a_{r}(t),
$$

where $\Gamma$ is a net-loss coefficient; $\phi, \beta_{1}$ are the phase and group-velocity delays, respectively; $\beta_{\mathrm{m}}$ are the $m$ th-order GDD coefficients, and $\gamma$ is the SPM coefficient.

In the absence of group-delay, GDD and SPM in ER, the energy, and power enhancement factors $\left(Q_{e}\right.$ and $Q_{p}$, respectively) are [87]:

$$
Q_{e}=Q_{p}=\left(\frac{\theta}{1-\operatorname{rexp}[-\Gamma]}\right)^{2} .
$$

Under the condition of weak changes of the field during single cavity round-trip, Eqs. (22) and (23) can be rewritten in the form of the generalized driven nonlinear Schrödinger equation for the intracavity field $a(t)[88]$ :

$$
\frac{\partial a(z, t)}{\partial z}=\left[-\Pi+i \Delta \phi-\beta_{1} \frac{\partial}{\partial t}+i \sum_{m=2} i^{m} \beta_{m} \frac{\partial^{m}}{\partial t^{m}}+i \gamma|a|^{2}\right] a+\theta \Phi(t),
$$

where $z$ is a cavity round-trip number, $\Pi=1-r+r \Gamma, \Phi(t)$ is an incident field amplitude and $\Delta \phi$ is a phase offset from the resonance $\phi=\pi$. In the absence of SPM (vacuum ER) but with GDD induced by resonator mirrors, the solutions for the energy and power enhancement factors are ${ }^{7}$ :

$$
\begin{aligned}
& Q_{e}=\frac{1}{2 \pi \int_{-\infty}^{\infty}|\Phi(t)|^{2} d t} \int_{-\infty}^{\infty}\left|\frac{\int_{-\infty}^{\infty} \Phi(t) e^{i \omega t}}{\Pi+i\left(\beta_{1} \omega-\beta_{2} \omega^{2}+\beta_{3} \omega^{3}-\beta_{4} \omega^{4}\right)}\right|^{2} d \omega, \\
& Q_{p}=\max \left\{\left|\frac{1}{2 \pi} \int_{-\infty}^{\infty}\left[\frac{\int_{-\infty}^{\infty} \Phi(t) e^{i \omega t} d t}{\Pi+i\left(\beta_{1} \omega-\beta_{2} \omega^{2}+\beta_{3} \omega^{3}-\beta_{4} \omega^{4}\right)}\right] e^{-i \omega t} d \omega\right|^{2}\right\} .
\end{aligned}
$$

\footnotetext{
${ }^{7} \Gamma \ll 1$ and $m \leq 4$ are assumed. The field amplitude and the pulse width are normalized to those of incident.
} 

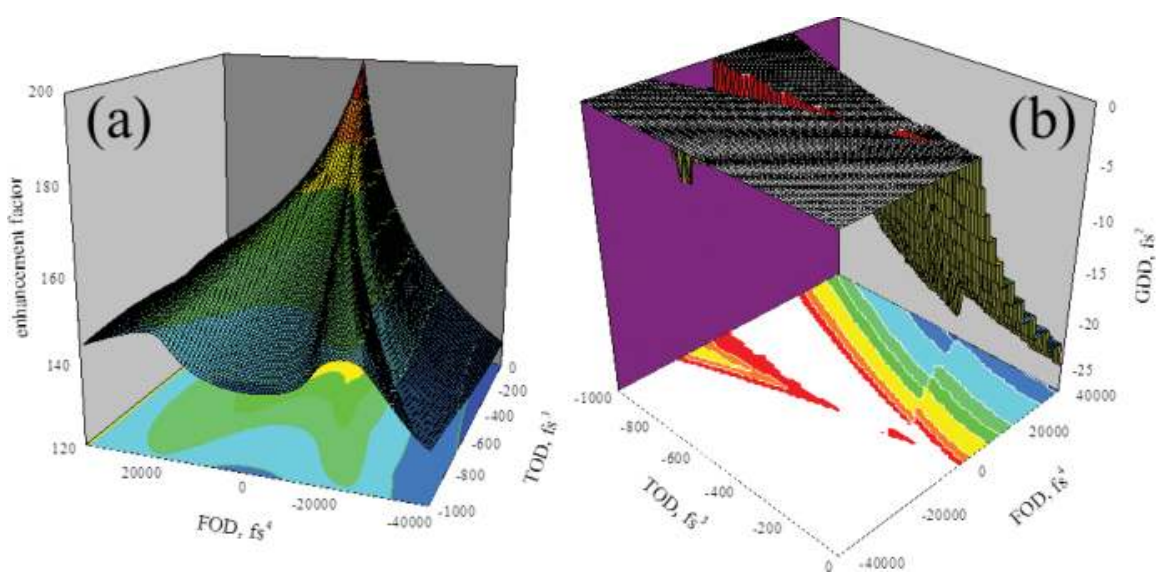

Figure 10. (a) Maximum $Q_{p}$ inside ER in the dependence of the third-order dispersion (TOD, $m=3$ ) and the fourth-order dispersion (FOD, $m=4$ ) for $\gamma=0, \Delta \phi=0, \beta_{1} \sim-0.5 \mathrm{fs}, \Gamma=0.5 \%$ and $25 \mathrm{fs}$ sech-shaped incident pulse at $790 \mathrm{~nm}$ central wavelength. (b) The corresponding optimal second-order dispersion (GDD, $m=2$ ) [88].

Eq. (26) demonstrates reducing the enhancement factors due to GDD [87]. Indeed, $\beta_{m} \neq 0$ reduces the spectral power at the spectrum edges $\propto 1 /\left(1+\omega^{2 m} \beta_{m}^{2}\right)$ that worsens the spectral overlap between the pulses from a laser and ER. In combination with a chirp appearance and a pulse broadening in ER, these factors drop both $Q_{e}$ and $Q_{p}$ (Figure 10).

It is clear that destructive action of higher-order GDD ( $m>2$ in Eq. (25)) on enhancement factor of ER grows with the pulse shortening so that a thorough dispersion-engineering of ER mirrors within a sufficiently broad spectral range is required $[28,87,88]$.

Additionally, the enhancement factor control can be provided by realizing a soliton-like regime in the nonlinear ER with $\gamma \neq 0$ (Figure 11) [87, 88]. In the absence of higher-order dispersions (i.e., $m=2)$, Eq. (25) has an exact soliton-like solution $a(t)=\sqrt{P(t)} \exp ($ iqz) :

$$
P(t)=\left(\frac{\theta}{\Pi}\right)^{2} \operatorname{sech}\left(\frac{t}{T}\right)^{2}, \beta_{2}=-\left(\frac{\theta T}{\Pi}\right)^{2} \frac{\gamma P(0)}{2}, \Delta \phi=-\left(\frac{\theta}{\Pi}\right)^{2} \frac{\gamma P(0)}{2}, q=0 .
$$

${ }^{8}$ This soliton-like pulse can be perturbed by higher-order dispersions $(m>2)$ induced by the broad-band ER mirrors so that optimization of ER parameters is required in this case, as well (Figure 11).

A promising possibility of the $Q_{p}$-increase results from a loss compensation by a gain inside ER. In this case, Eq. (25) has to be supplemented by the term $r \rho \tau \partial_{t, t} a$ with the modified $\Pi=1-r+r(\Gamma-\rho)^{9}$ (see Eqs. (9) and (25)). One has to note, that ER remains below lasing threshold and a resonator mode in an active crystal (Ti: sapphire in our case) has to be sufficiently broad to suppress gain

${ }^{8}$ Here $\beta_{2}<0$ corresponds to an anomalous dispersion. $\stackrel{9}{\rho}<\Gamma$ is the stability condition. 

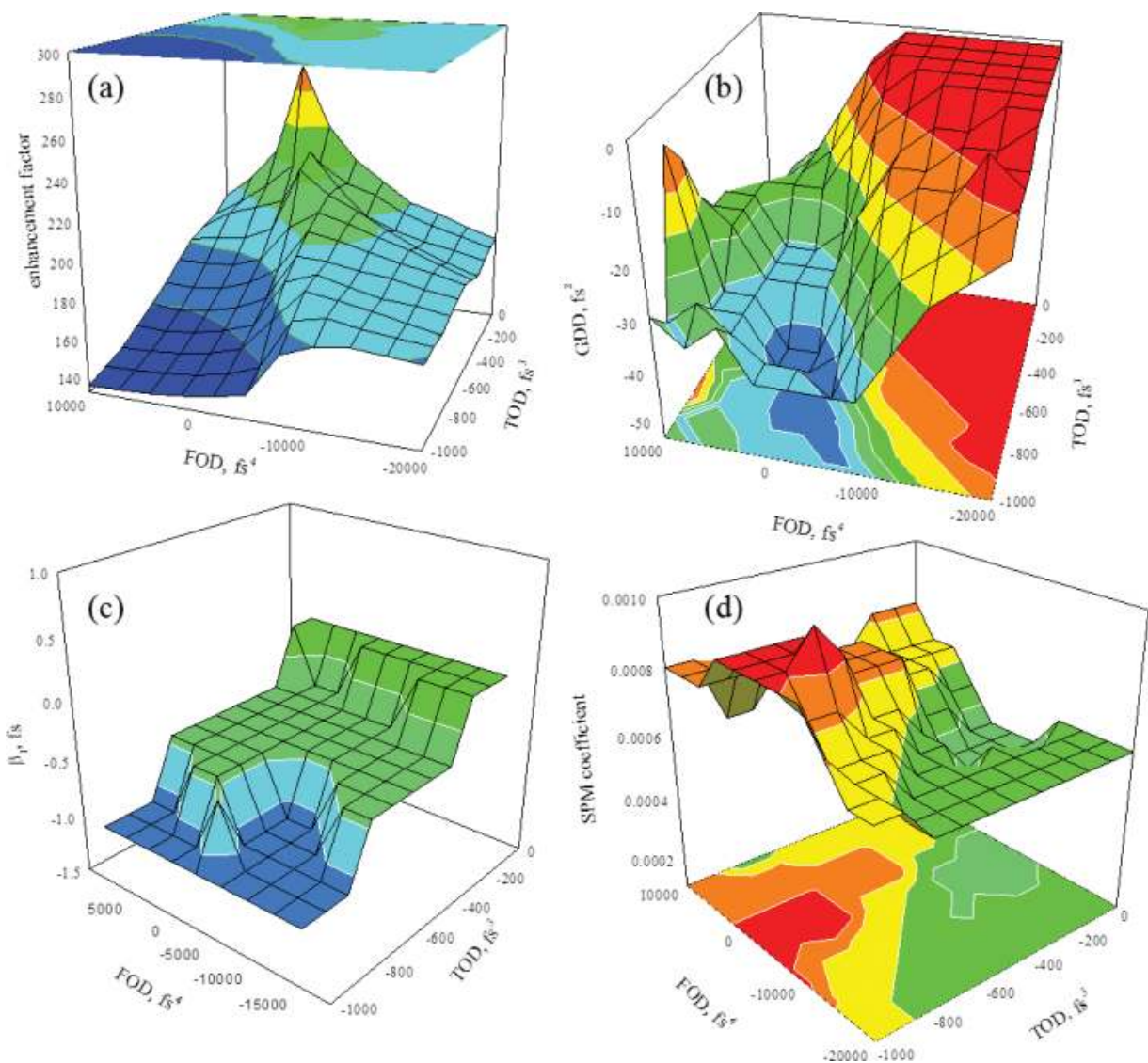

Figure 11. Dependence of the maximum $Q_{p}$ on TOD and FOD (a) optimized by control of $\beta_{1}$ (c), $\beta_{2}$ (b), and the dimensionless SPM coefficient $\gamma P_{\max }(\mathrm{d})$. Incident pulse $T_{\mathrm{FWHM}}$ is $25 \mathrm{fs}, \Gamma=0.25 \%$.
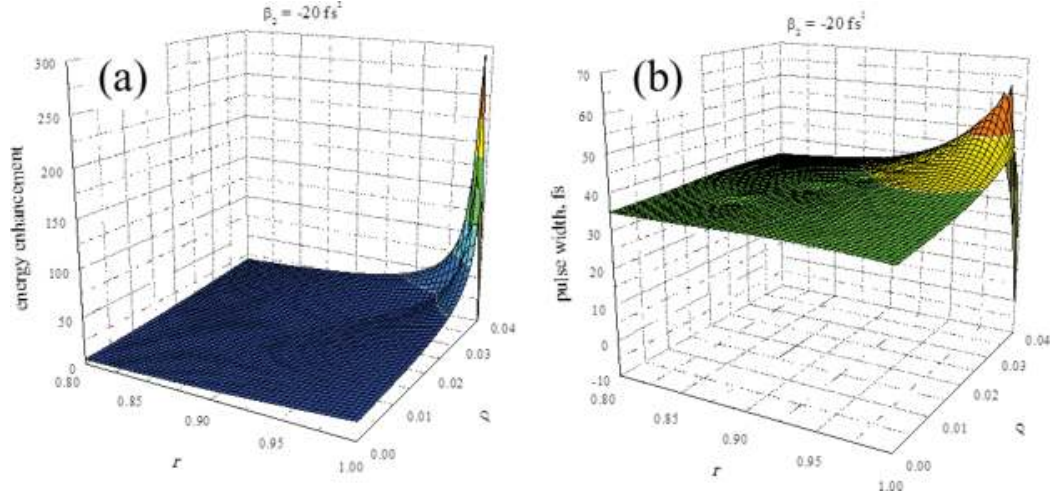

Figure 12. $Q_{e}$ (a) and the pulse width (b) in the presence of gain, SPM, and GDD in ER. $\Gamma=0.04$, the width of the incident $100 \mathrm{~nJ}$ pulse is of $30 \mathrm{fs}$, the laser beam size in the $2 \mathrm{~mm}$ Ti: sapphire active crystal is $1.1 \mathrm{~mm}$. 
saturation and SPM. The soliton-like regime increases the enhancement factor and reduces the sensitivity to higher-order dispersions in this case, as well (Figure 12).

\section{Conclusions}

In this work, the approaches to an energy harvesting at femtosecond scale are reviewed and elaborated theoretically with a close connection with both solid-state and fiber ML oscillators including, in particular, a nonlinear ER. The basic concept here is a dissipative soliton allowing an extra-energy and spectral width scaling under fine control of the laser parameters. This concept is a very productive for different applications and brings a high-energy physics in "physics laboratory" where extremal parameters result from not an onslaught but rather "subtle tuning." This tuning requires multi-disciplinary approaches providing the multi-scale power and energy harvesting, which application areas range from fundamental quantum mechanics to neuroscience and sociology, and include, in particular, a "quantum engineering" of Bose-Einstein and quasi-particle condensates. A further outlook concerns a study of nonlinear dynamics of complicated nonlinear systems far from thermodynamic equilibrium, which is based on their "metaphoric" modeling in more simple and controllable laser systems.

\section{Acknowledgements}

The author acknowledges the support from Austrian Science Fund (FWF project P24916). Computational results have been achieved using the Vienna Scientific Cluster (VSC).

\section{Author details}

Vladimir L. Kalashnikov

Address all correspondence to: vladimir.kalashnikov@tuwien.ac.at

Institute of Photonics, Vienna University of Technology, Vienna, Austria

\section{References}

[1] Südmeyer T, Marchese SV, Hashimoto S, Baer CRE, Gingras G, Witzel B, Keller U. Femtosecond laser oscillators for high-field science. Nature Photonics. 2008;2:599-604. DOI: 10.1038/nphoton.2008.194

[2] Brabec T, editor. Strong Field Laser Physics. New York: Springer; 2008. 585 p. DOI: 10.1007/ 978-0-387-34755-4 
[3] Marchese SV, Baer CRE, Engqvist AG, Hashimoto S, Maas DJHC, Golling M, Südmeyer T, Keller U. Femtosecond thin disk laser oscillator with pulse energy beyond the 10-microjoule level. Optics Express. 2008;16(9):6397-6407. DOI: 10.1364/OE.16.006397

[4] Bauer D, Zawischa I, Sutter DH, Killi A, Dekorsy T. Mode-locked Yb:YAG thin-disk oscillator with $41 \mu \mathrm{J}$ pulse energy at $145 \mathrm{~W}$ average infrared power and high power frequency conversion. Optics Express. 2012;20(9):9698-9704. DOI: 10.1364/OE.20.009698

[5] Saraceno CJ, Emaury F, Schriber C, Hoffmann M, Golling M, Südmeyer T, Keller U. Ultrafast thin-disk laser with $80 \mu \mathrm{J}$ pulse energy and $242 \mathrm{~W}$ of average power. Optics Letters. 2014;39(1):9-12. DOI: 10.1364/OL.39.000009

[6] Saraceno CJ, Emaury F, Heckl OH, Baer CRE, Hoffmann M, Schriber C, Golling M, Südmeyer T, Keller U. 275 W average output power from a femtosecond thin disk oscillator operated in a vacuum environment. Optics Express. 2012;20(21):23535-23541. DOI: 10.1364/OE.20.023535

[7] Brons J, Pervak V, Fedulova E, Bauer D, Sutter D, Kalashnikov V, Apolonskiy A, Pronin O, Krausz F. Energy scaling of Kerr-lens mode-locked thin-disk oscillators. Optics Letters. 2014;39(22):6442-6445. DOI: 10.1364/OL.39.006442

[8] Emaury F, Diebold A, Saraceno CJ, Keller U. Compact extreme ultraviolet source at megahertz pulse repetition rate with a low-noise ultrafast thin-disk laser oscillator. Optica. 2015;2(11):980-984. DOI: 10.1364/OPTICA.2.000980

[9] Backus S, Durfee CG III, Murnane MM, Kapteyn HC. High power ultrafast lasers. Review of Scientific Instruments. 1998;69(3):1207-1223

[10] Mourou GA, Tajima T, Bulanov SV. Optics in the relativistic regime. Reviews of Modern Physics. 2006;78(2):309-371. DOI: 10.1103/RevModPhys.78.309

[11] Liu Y, Tschuch S, Rudenko A, Dürr M, Siegel M, Morgner U, Moshammer R, Ullrich J. Strong-field double ionization of Ar below the recollision threshold. Physical Review Letters. 2008;101:053001. DOI: 10.1103/PhysRevLett.101.053001

[12] Seres E, Seres J, Spielmann C. Extreme ultraviolet light source based on intracavity high harmonic generation in a mode locked Ti:sapphire oscillator with $9.4 \mathrm{MHz}$ repetition rate. Optics Express. 2012;20(6):6185-6190. DOI: 10.1364/OE.20.006185

[13] Sciaini G, Miller RJD. Femtosecond electron diffraction: Heralding the era of atomically resolved dynamics. Reports on Progress in Physics. 2011;74:096101. DOI: 10.1088/00344885/74/9/096101

[14] Gattass RR, Mazur E. Femtosecond laser micromachining in transparent materials. Nature Photonics. 2008;2:219-225. DOI: 10.1038/nphoton.2008.47

[15] Lanin AA, Fedotov IV, Sidorov-Biryukov DA, Doronina-Amitonova LV, Ivashkina OI, Zots MA, Sun C-K, Ilday FÖ, Fedotov AB, Anokhin KV, Zheltikov AM. Air-guided photonic-crystal-fiber pulse-compression delivery of multimegawatt femtosecond laser 
output for nonlinear-optical imaging and neurosurgery. Applied Physics Letters. 2012; 100:101104. DOI: 10.1063/1.3681777

[16] Gallmann L, Cirelli C, Keller U. Attosecond science: Recent highlights and future trends. Annual Review of Physical Chemistry. 2012;63:447-469. DOI: 10.1146/annurev-physchem032511-143702

[17] Akhmediev NN, Ankiewicz A, editors. Dissipative Soliton. Berlin: Springer; 2005. 456 p. DOI: $10.1007 / \mathrm{b} 11728$

[18] Spatschek KH, Mertens FG, editors. Nonlinear Coherent Structures in Physics and Biology. New York: Springer; 1994. 476 p. DOI: 10.1007/978-1-4899-1343-2

[19] Dawes JHP. The emergence of a coherent structure for coherent structures: Localized states in nonlinear systems. Philosophical Transactions of the Royal Society A. 2010;368: 3519-3534. DOI: 10.1098/rsta.2010.0057

[20] Kevrekidis PG, Frantzeskakis DJ, Carretero-Gonzalez R, editors. Emergent Nonlinear Phenomena in Bose-Einstein Condensates. Berlin: Springer; 2008. 385 p

[21] Editorial. The power of analogues. Nature Photonics. 2014;8:1. DOI: 10.1038/nphoton. 2013.359

[22] Scott AC. The Nonlinear Universe: Chaos, Emergence, Life. Berlin: Springer; 2010. 364 p

[23] Ph G, Akhmediev N. Dissipative solitons for mode-locked lasers. Nature Photonics. 2012; 6:84-92. DOI: 10.1038/NPHOTON.2011.345

[24] Kalashnikov VL. Chirped-pulse oscillators: Route to the energy scalable femtosecond pulses. In: Al-Khursan AH, editor. Solid-State Laser. Rijeka: InTechOpen; 2012. pp. 145184. DOI: $10.5772 / 37415$

[25] Kalashnikov VL, Sergeyev SV. Dissipative solitons in fibre lasers. In: Paul MC, editor. Fibre Laser. Rijeka: InTechOpen; 2016. pp. 165-210. DOI: 10.5772/61713

[26] Ashkin A, Boyd G, Dziedzic J. Resonant optical second harmonic generation and mixing. IEEE Journal of Quantum Electronics. 1966;2(6):109-124. DOI: 10.1109/JQE.1966.1074007

[27] Yanovsky VP, Wise FW. Frequency doubling of 100-fs pulses with 50\% efficiency by use of a resonant enhancement cavity. Optics Letters. 1994;19(23):1952-1954. DOI: 10.1364/ OL.19.001952

[28] Lilienfein N, Hofer C, Holzberger S, Matzer C, Zimmermann P, Trubetskov M, Pervak V, Pupeza I. Enhancement cavities for few-cycle pulses. Optics Letters. 2017;42(2):271-274. DOI: $10.1364 /$ OL.42.000271

[29] Siegman AE. Lasers. Mill Valley: University Science Books; 1986. 1283 p

[30] Kalashnikov VL. Mathematical Ultrashort-Pulse Laser Physics [Internet]. March 26, 2002. Available from: https://www.maplesoft.com/applications/view.aspx?SID=4247 
[31] Abraham NB, Lugiato LA, Narducci LM. Overview of instabilities in laser systems. Journal of the Optical Society of America B: Optical Physics. 1985;2(1):7-14. DOI: 10.1364/ JOSAB.2.000007

[32] Goodberlet J, Wang J, Fujimoto JG, Schulz PA. Starting dynamics of additive-pulse mode locking in the Ti: $\mathrm{Al}_{2} \mathrm{O}_{3}$ laser. Optics Letters. 1990;15(22):1300-1302. DOI: 10.1364/OL.15.001300

[33] Krausz F, Fermann ME, Brabec T, Curley PF, Hofer M, Ober MH, Spielmann C, Wintner E, Schmidt AJ. Femtosecond solid-state lasers. IEEE Journal of Quantum Electronics. 1992;28(10):2097-2122. DOI: 10.1109/3.159520

[34] Krausz F, Brabec T. Passive mode locking in standing-wave laser resonators. Optics Letters. 1993;18(11):888-890. DOI: 10.1364/OL.18.000888

[35] Ippen EP, Liu LY, Haus HA. Self-starting condition for additive-pulse mode-locked lasers. Optics Letters. 1990;15(3):183-185. DOI: 10.1364/OL.15.000183

[36] Komarov AK, Komarov KP, Mitschke FM. Phase-modulation bistability and threshold self-start of laser passive mode locking. Physical Review A. 2002;65:053803. DOI: 10.1103/ PhysRevA.65.053803

[37] Chen C-J, Wai PKA, Menyuk CR. Self-starting of passively mode-locked lasers with fast saturable absorbers. Optics Letters. 1995;20(4):350-352. DOI: 10.1364/OL.20.000350

[38] Soto-Crespo JM, Akhmediev N, Town G. Continuous-wave versus pulse regime in a passively mode-locked laser with a fast saturable absorber. Journal of the Optical Society of America B. 2002;19(2):234-242. DOI: 10.1364/JOSAB.19.000234

[39] Risken H, Nummedal K. Self-pulsing in lasers. Journal of Applied Physics. 1968;39(10): 4662-4672. DOI: 10.1063/1.1655817

[40] Gordon A, Fischer B. Phase transition theory of pulse formation in passively mode-locked lasers with dispersion and Kerr nonlinearity. Optics Communications. 2003;223:151-156. DOI: 10.1016/S0030-4018(03)01622-5

[41] Vodonos B, Bekker A, Smulakovsky V, Gordon A, Gat O, Berger NK, Fischer B. Experimental study of the stochastic nature of the pulsation self-starting process in passive mode locking. Optics Letters. 2005;30(20):2787-2789. DOI: 10.1364/OL.30.002787

[42] Gordon A, Gat O, Fischer B, Kärtner FX. Self-starting of passive mode locking. Optics Express. 2006;14(23):11142-11154. DOI: 10.1364/OE.14.011142

[43] Gammaitoni L, Hänggi P, Jung P, Marchesoni F. Stochastic resonance. Reviews of Modern Physics. 1998;70(1):223-287

[44] Kivshar YS, Agrawal GP. Optical Solitons: From Fibers to Photonic Crystals. Amsterdam: Academic Press; 2003. 540 p

[45] Aranson IS, Kramer L. The world of the complex Ginzburg-Landau equation. Reviews of Modern Physics. 2002;79(1):99-143. DOI: 10.1103/RevModPhys.74.99 
[46] García-Morales V, Krischer K. The complex Ginzburg-Landau equation: An introduction. Contemporary Physics. 2012;53(2):79-95. DOI: 10.1080/00107514.2011.642554

[47] van Saarloos W, Hohenberg PC. Fronts, pulses, sources and sinks in generalized complex Ginzburg-Landau equations. Physica D. 1992;56:303-367

[48] Akhmediev NN, Ankiewicz A, editors. Dissipative Solitons: from Optics to Biology and Medicine. Berlin: Springer; 2008

[49] Anderson D, Lisak M, Berntson A. A variational approach to nonlinear evolution equations in optics. Pramana-Journal of Physics. 2001;57(5-6):917-936. DOI: 10.1007/s12043001-0006-z

[50] Malomed BA. Variational methods in nonlinear fiber optics and related fields. In: Wolf E, editor. Progress in Optics. Amsterdam: Elsevier; 2002. pp. 71-193

[51] Ankiewicz A, Akhmediev N, Devine N. Dissipative solitons with a Lagrangian approach. Optical Fiber Technology. 2007;13(2):91-97. DOI: 10.1016/j.yofte.2006.12.001

[52] Kalashnikov VL. Chirp-free Solitons in Dissipative Systems: Variational Approximation and Issue of the Soliton Energy Scalability [Internet]. May 30, 2014. Available from: http:// info.tuwien.ac.at/kalashnikov/variational_soliton.pdf

[53] Kantorovich LV, Krylov VI. Approximate Methods of Higher Analysis. Groningen: P. Noordhoff Ltd; $1958.681 \mathrm{p}$

[54] Cerda SC, Cavalcanti SB, Hickmann JM. A variational approach to nonlinear dissipative pulse propagation. European Physical Journal D: Atomic, Molecular, Optical and Plasma Physics. 1998;1:313-316

[55] Kalashnikov VL, Sorokin E, Sorokina IT. Multipulse operation and limits of the Kerr-lens mode locking stability. IEEE Journal of Quantum Electronics. 2003;39(2):323-336. DOI: 10.1109/JQE.2002.807204

[56] Vakhitov NG, Kolokolov AA. Stationary solutions of the wave equation in a medium with nonlinearity saturation. Radiophysics and Quantum Electronics. 1973;16(7):783-789

[57] Bergé L. Wave collapse in physics: Principles and applications to light and plasma waves. Physics Reports. 1998;303(5-6):259-370. DOI: 10.1016/S0370-1573(97)00092-6

[58] Baer CRE, Heckl OH, Saraceno CJ, Schriber C, Kränkel C, Südmeyer T, Keller U. Frontiers in passively mode-locked high-power thin disk laser oscillators. Optics Express. 2012; 20(7):7054-7065. DOI: 10.1364/OE.20.007054

[59] Saraceno CJ, Heckl OH, Baer CRE, Schriber C, Golling M, Beil K, Kränkel C, Südmeyer T, Huber G, Keller U. Sub-100 femtosecond pulses from a SESAM modelocked thin disk laser. Applied Physics B. 2012;106(3):559-562. DOI: 10.1007/s00340-012-4900-5

[60] Paré C, Bélanger P-A. Optical solitary waves in the presence of a Lorentzian gain line: Limitations of the Ginzburg-Landau model. Optics Communications. 1998;145(1-6):385392. DOI: 10.1016/S0030-4018(97)00433-1 
[61] Kalashnikov VL, Apolonski A. Energy scalability of mode-locked oscillators: A completely analytical approach to analysis. Optics Express. 2010;18(25):25757-25770. DOI: 10.1364/ OE.18.025757

[62] Zhang J, Brons J, Seidel M, Pervak V, Kalashnikov V, Wei Zh, Apolonski A, Krausz F, Pronin O. 49-fs Yb: YAG thin-disk oscillator with distributed Kerr-lens mode-locking. In: European Conference on Lasers and Electro-Optics-European Quantum Electronics Conference; 21-25 June 2015; Munich, Germany. Optical Society of America; 2015. p. PD_A_1

[63] Podivilov E, Kalashnikov VL. Heavily-chirped solitary pulses in the normal dispersion region: New solutions of the cubic-quintic complex Ginzburg-Landau equation. JETP Letters. 2005;82(8):467-471

[64] Kalashnikov VL. Chirped-pulse oscillators: A unified standpoint. Physical Review A. 2009;79:043829. DOI: 10.1103/PhysRevA.79.043829

[65] Chang W, Ankiewicz A, Soto-Crespo JM, Akhmediev N. Dissipative soliton resonances. Physical Review A. 2008;78:023830. DOI: 10.1103/PhysRevA.78.023830

[66] Kalashnikov VL. Chirped Solitary-Pulse Solutions of the Complex Generalized Nonlinear Ginzburg-Landau Equation (II) [Internet]. February 2, 2009. Available from: http://info. tuwien.ac.at/kalashnikov/genNCGLE.html

[67] Kalashnikov VL, Sorokin E, Sorokina IT. Chirped dissipative soliton absorption spectroscopy. Optics Express. 2011;19(18):17480-17492

[68] Zhu L, Verhoef AJ, Jespersen KG, Kalashnikov VL, Grüner-Nielsen L, Lorenc D, Baltuška A, Fernández A. Generation of high fidelity 62-fs, 7-nJ pulses at $1035 \mathrm{~nm}$ from a net normal-dispersion $\mathrm{Yb}$-fiber laser with anomalous dispersion higher-order-mode fiber. Optics Express. 2013;21(14):16255-16262. DOI: 10.1364/OE.21.016255

[69] Pronin O, Brons J, Grasse C, Pervak V, Boehm G, Amann M-C, Apolonski A, Kalashnikov VL, Krausz F. High-power Kerr-lens mode-locked Yb:YAG thin-disk oscillator in the positive dispersion regime. Optics Letters. 2012;37(17):3543-3545. DOI: 10.1364/OL.37.003543

[70] Li H, Ouzounov DG, Wise FW. Starting dynamics of dissipative-soliton fiber laser. Optics Letters. 2010;35(14):2403-2405. DOI: 10.1364/OL.35.002403

[71] Kalashnikov VL, Kalosha VP, Poloiko IG, Mikhailov VP. New principle of formation of ultrashort pulses in solid-state lasers with self-phase-modulation and gain saturation. Quantum Electronics. 1996;26(3):236-239

[72] Connaughton C, Josserand C, Picozzi A, Pomeau Y, Rica S. Condensation of classical nonlinear waves. Physical Review Letters. 2005;95:263901. DOI: 10.1103/PhysRevLett.95. 263901

[73] Michel C, Kibler B, Picozzi A. Discrete spectral incoherent solitons in nonlinear media with noninstantaneous response. Physical Review A. 2011;83:023806. DOI: 10.1103/ PhysRevA.83.023806 
[74] Kalashnikov VL. Optics and chaos: Chaotic, rogue, and noisy optical dissipative solitons. In: Skiadas CH, Skiadas C, editors. Handbook of Applications of Chaos Theory. London: CRC Press; 2016. pp. 587-626

[75] Kalashnikov VL, Podivilov E, Chernykh A, Naumov S, Fernandez A, Graf R, Apolonski A. Approaching the microjoule frontier with femtosecond laser oscillators: Theory and comparison with experiment. New Journal of Physics. 2005;7:217. DOI: 10.1088/1367-2630/7/1/217

[76] Kalashnikov VL, Podivilov E, Chernykh A, Apolonski A. Chirped-pulse oscillators: Theory and experiment. Applied Physics B. 2006;83:503-510. DOI: 10.1007/s00340-006-2214-1

[77] Kalashnikov VL. Chirped-Pulse Oscillators: An Impact of the Dynamic Gain Saturation [Internet]. July 7, 2008. Available from: https://arxiv.org/abs/0807.1050

[78] Kharenko DS, Podivilov EV, Apolonski AA, Babin SA. 20 nJ 200 fs all-fiber highly chirped dissipative soliton oscillator. Optics Letters. 2012;37(19):4104-4106. DOI: 10.1364/OL.37. 004104

[79] Babin SA, Podivilov EV, Kharenko DS, Bednyakova AE, Fedoruk MP, Kalashnikov VL, Apolonski A. Multicolour nonlinearly bound chirped dissipative solitons. Nature Communications. 2014;5:4653. DOI: 10.1038/ncomms5653

[80] Kalashnikov VL, Evgeni S. Dissipative Raman solitons. Optics Express. 2014;22(24): 30118-30126. DOI: 10.1364/OE.22.030118

[81] Babin SA, Podivilov EV, Kharenko DS, Bednyakova AE, Fedoruk MP, Shtyrina OV, Kalashnikov VL, Apolonski AA. SRS-driven evolution of dissipative solitons in fiber lasers. In: Ph G, editor. Nonlinear Optical Cavity Dynamics: From Microresonators to Fiber Lasers. Mannheim: Wiley; 2016. pp. 277-316. DOI: 10.1002/9783527686476.ch12

[82] Kalashnikov VL, Sorokin E. Turbulence of optical dissipative solitons. In: Skiadas ChH, editor. Book of Abstracts of 10th Chaotic Modeling and Simulation International Conference; 30 May-2 June, 2017; Barcelona, Spain. ISAST: International Society for the Advancement of Science and Technology; 2017. p. 64

[83] Bernhardt B, Ozawa A, Jacquet P, Jacquey M, Kobayashi Y, Udem T, Holzwarth R, Guelachvili G, Hänsch TW, Picqué N. Cavity-enhanced dual-comb spectroscopy. Nature Photonics. 2010;4:55-57. DOI: 10.1038/nphoton.2009.217

[84] Pupeza I, Holzberger S, Eidam T, Carstens H, Esser D, Weitenberg J, Rußbüldt P, Rauschenberger J, Limpert J, Udem T, Tünnermann A, Hänsch TW, Apolonski A, Krausz F, Fill E. Compact high-repetition-rate source of coherent $100 \mathrm{eV}$ radiation. Nature Photonics. 2013;7:608-612. DOI: 10.1038/nphoton.2013.156

[85] Carstens H, Högner M, Saule T, Holzberger S, Lilienfein N, Guggenmos A, Jocher C, Eidam T, Esser D, Tosa V, Pervak V, Limpert J, Tünnermann A, Kleineberg U, Krausz F, Pupeza I. High-harmonic generation at $250 \mathrm{MHz}$ with photon energies exceeding $100 \mathrm{eV}$. Optica. 2016;3(4):366-369. DOI: 10.1364/OPTICA.3.000366

[86] Ippen EP, Haus HA, Liu LY. Additive pulse mode locking. Journal of the Optical Society of America B. 1989;6(9):1736-1745. DOI: 10.1364/JOSAB.6.001736 
[87] Kalashnikov VL. Femtosecond pulse enhancement in an external resonator: Impact of dispersive and nonlinear effects. Applied Physics B: Lasers and Optics. 2008;92:19-23. DOI: 10.1007/s00340-008-3064-9

[88] Kalashnikov VL, Gohle Ch, Udem Th. Maximization of ultrashort pulse power stored in a passive resonator synchronously pumped by a femtosecond oscillator. In: Denman C, Sorokina I, editors. Advanced Solid-State Photonics (TOPS); February 6-9, 2005; Vienna, Austria. Optical Society of America; 2005. p. 652. DOI: 10.1364/ASSP.2005.652 